\section{Routine, single session, indirect laser for proliferative diabetic retinopathy}

\begin{abstract}
Aims To evaluate the visual and morphological outcomes of routine, single session, indirect panretinal photocoagulation (PRP) for proliferative diabetic retinopathy, and to examine adverse events related to indirect laser within the first 8 weeks of treatment.
\end{abstract}

Methods The case notes of 107 diabetics with proliferative retinopathy undergoing indirect PRP were reviewed retrospectively, and compared with the UK National Diabetic Retinopathy Laser Treatment Audit. Patients who had received PRP previously were excluded. Follow-up data were collected as close as possible to 9 months following the initial laser treatment.

Results Fifteen patients (14.0\%) returned with adverse events within the first 8 weeks of indirect PRP. There were two tractional retinal detachments and two new vitreous haemorrhages. One patient with underlying neurotrophic keratopathy developed a persistent epithelial defect. One developed non-progressive macular drag with a one-line drop in Snellen acuity. Three experienced new or exacerbations of preexisting clinically significant macular oedema, which resolved spontaneously. There were two transient choroidal effusions, two transient anterior uveitis, and two transient visual deteriorations without macular oedema clinically. There were no significant differences in the rates of new tractional retinal detachment, vitreous haemorrhage or rubeosis, but our vitrectomy rate was higher $\left(7.5\right.$ vs $\left.1.5 \%, P=0.02, \chi^{2}\right)$, largely due to our policy of performing vitrectomy early.

Conclusions Visual and morphological outcomes of routine, single session, indirect PRP for proliferative diabetic retinopathy
CG Tinley and RH Gray

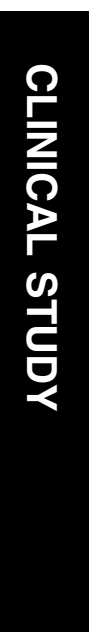

were not inferior to the outpatient-based national audit, and the incidence of significant PRP-induced adverse events was low. Eye (2009) 23, 1819-1823; doi:10.1038/eye.2008.394; published online 9 January 2009

Keywords: single session; indirect; laser; proliferative; diabetic; retinopathy

\section{Introduction}

The binocular indirect argon laser photocoagulator was first described by Mizuno ${ }^{1}$ in 1981, who suggested it might become the ideal method for panretinal photocoagulation (PRP) in the treatment of diabetic retinopathy. Currently, slit-lamp delivery of laser remains standard practice in the management of diabetics with proliferative retinopathy. In many centres, the use of indirect laser is limited to patients with physical, medical, or mental handicaps that preclude slit-lamp treatment, or to patients where progression of severe retinopathy is a concern. ${ }^{2}$ However, we routinely perform indirect PRP in all patients with proliferative diabetic retinopathy (PDR), as we believe that it offers easier access to the far retinal periphery, and improved patient comfort. It is intended that PRP should be completed in one session under local anaesthetic block for unilateral disease or under general anaesthesia (GA) if both eyes require treatment. If retinopathy progresses after PRP, with signs of vitreous haemorrhage $(\mathrm{VH})$ or tractional retinal detachment (TRD), an early vitrectomy is performed. PRP can be a painful procedure for many patients, and when performed under peribulbar block, it is significantly less painful than topical anaesthesia, even when augmented with
Department of

Ophthalmology, Taunton and Somerset NHS Trust, Musgrove Park Hospital, Somerset, UK Department of Ophthalmology, Taunton and Somerset NHS Trust,

Musgrove Park Hospital, Taunton,

Somerset TA1 5DA, UK Tel: + 441823 342942;

Fax: + 441823342943

E-mail: roger.gray@

tst.nhs.uk

Received: 15 May 2008 Accepted in revised form: 28 November 2008

Published online: 9 January 2009

This study was previously presented at South Western Ophthalmological Society Meeting, 2007

Proprietary interest/research funding: None
Correspondence: RH Gray, 
intramuscular or oral analgesics. ${ }^{3}$ Better tolerance of the procedure also allows for quicker and more complete treatment. The perceived disadvantage of performing complete PRP in one session is the possible increase in complications, such as macular oedema and choroidal effusions. The primary aims of this study were to compare the visual and morphological outcomes of routine, single session, indirect PRP for PDR with the UK National Diabetic Retinopathy Laser Treatment Audit (NDRLTA), ${ }^{4,5}$ and to examine the adverse events related to indirect PRP within the first 8 weeks of treatment.

\section{Materials and methods}

Surgical records were used to identify patients with PDR undergoing indirect PRP in theatre at Musgrove Park Hospital (MPH), Taunton, UK, between 2000 and 2006. For those undergoing bilateral simultaneous treatment, the eye with the worse visual acuity (VA) was included. Patients who had received prior PRP treatment were excluded from the study. The case notes of 107 patients were reviewed retrospectively. All procedures were carried out under peribulbar local anaesthetic block or GA. Pupils were dilated with two drops of $2.5 \%$ phenylephrine and four drops of $1 \%$ cyclopentolate, and patients were also given two drops of $0.1 \%$ diclofenac. Indirect laser was performed using the Lumenis Novus 2000 machine and a 20 or $30 \mathrm{D}$ lens. Typical laser settings were $200-300 \mathrm{~mW}$ power on continuous mode, with burn duration adjusted to produce moderately intense burns, spaced approximately one burn width apart, and extending to the far retinal periphery. At the end of the procedure, the eye was padded and patients discharged on oral flurbiprofen $50 \mathrm{mg}$ tds for 2 weeks. Patient demographics and outcomes were compared with the UK NDRLTA, a prospective, multicentre survey published by the Royal College of Ophthalmologists in 1998. A proforma modelled on the NDRLTA was used to enter data on retinopathy features, best-recorded VA, amount of treatment given, and the grade of ophthalmologist performing the treatment. Final followup data were collected as close as possible to 9 months following the initial laser treatment and included VA, change in retinopathy features, and any follow-up treatment given. All information was stored in accordance with the Data Protection Act and analysed using a commercially available statistical software package (SSPS 14.0).

\section{Results}

A comparison of patient demographics and baseline characteristics can be found in Table 1. Best-recorded Snellen VAs pre- and post-treatment are presented in
Table 1 Baseline characteristics

\begin{tabular}{lcc}
\hline & MPH & NDRLTA \\
\hline$n$ & 107 & 284 \\
Mean age at PRP (years) & 54.5 & 54.8 \\
Age range (years) & $20-79$ & $17-85$ \\
Duration of diabetes at PRP (years) & $1-44$ & $1-55$ \\
Mean duration of diabetes (years) & 17.8 & 16.8 \\
& & \\
Female & $\%$ & $\%$ \\
Male & 29.0 & 42.1 \\
Type I diabetes & 71.0 & 57.9 \\
Type II diabetes & 35.5 & 31.2 \\
& 64.5 & 68.8 \\
Best-recorded Snellen visual acuity & & \\
$\quad$ 6/9 or better treated eye & \\
$\quad$ 6/24 or worse treated eye & \\
& & \\
Anaesthesia & 75.7 & 55.4 \\
$\quad$ Local block & 11.2 & 18.3 \\
$\quad$ General & & \\
$\quad$ Bilateral simultaneous PRP & 61.7 & 6.9 \\
$\quad$ Intention to complete PRP in one session & 100 & 41.2 \\
\hline
\end{tabular}

$\mathrm{MPH}=$ Musgrove Park Hospital; NDRLTA = National Diabetic Laser Treatment Audit; $\mathrm{PDR}=$ proliferative diabetic retinopathy; $\mathrm{PRP}=$ panretinal photocoagulation.

${ }^{\mathrm{a}} P<0.01, \chi^{2} ;{ }^{\mathrm{b}} P=0.12, \chi^{2}$.

Table 2 Best-recorded Snellen visual acuity before treatment and 9 months post-treatment ${ }^{\mathrm{a}}$

\begin{tabular}{|c|c|c|c|c|c|c|c|c|}
\hline \multirow[t]{3}{*}{$V A$} & \multicolumn{4}{|c|}{$M P H$} & \multicolumn{4}{|c|}{ NDRLTA } \\
\hline & \multicolumn{2}{|c|}{ Pre } & \multicolumn{2}{|c|}{ Post } & \multicolumn{2}{|c|}{ Pre } & \multicolumn{2}{|c|}{ Post } \\
\hline & $\mathrm{n}$ & $\%$ & $\mathrm{n}$ & $\%$ & $\mathrm{n}$ & $\%$ & $\mathrm{n}$ & $\%$ \\
\hline $6 / 5$ & 18 & 16.8 & 17 & 15.9 & 32 & 11.3 & 19 & 9.1 \\
\hline $6 / 6$ & 11 & 10.2 & 23 & 21.5 & 52 & 18.4 & 36 & 17.2 \\
\hline $6 / 9$ & 52 & 48.6 & 38 & 35.5 & 73 & 25.7 & 59 & 28.2 \\
\hline $6 / 12$ & 12 & 11.2 & 10 & 9.3 & 46 & 16.2 & 38 & 18.2 \\
\hline $6 / 18$ & 2 & 1.9 & 7 & 6.6 & 28 & 9.9 & 11 & 5.3 \\
\hline $6 / 24$ & 7 & 6.5 & 1 & 0.9 & 15 & 5.3 & 12 & 5.7 \\
\hline $6 / 36$ & 2 & 1.9 & 1 & 0.9 & 10 & 3.5 & 7 & 3.3 \\
\hline $6 / 60$ & 0 & 0 & 3 & 2.8 & 6 & 2.1 & 9 & 4.3 \\
\hline$<6 / 60$ & 3 & 2.8 & 7 & 6.6 & 21 & 7.4 & 18 & 8.6 \\
\hline Total & 107 & 100 & 107 & 100 & 283 & 100 & 209 & 100 \\
\hline
\end{tabular}

$\mathrm{MPH}=$ Musgrove Park Hospital; NDRLTA = National Diabetic Retinopathy Laser Treatment Audit.

${ }^{a}$ The best-recorded visual acuity using spectacles and/or pinhole if required.

Table 2. At the time of laser, 16 patients $(15.0 \%)$ had concurrent clinically significant macular oedema (CSMO). Nine of these $(56.3 \%)$ received focal laser treatment before PRP, whereas two $(12.5 \%)$ were treated after PRP. In three cases (18.7\%), no laser treatment was planned, and in a further two, laser was not performed due to preexisting macular ischaemia. 
Table 3 Adverse events occurring within 8 weeks of PRP

\begin{tabular}{lcc}
\hline & $\mathrm{n}$ & $\%$ \\
\hline Tractional retinal detachment & 2 & 1.9 \\
New vitreous haemorrhage & 2 & 1.9 \\
Persistent corneal epithelial defect & 1 & 0.9 \\
Macular drag & 1 & 0.9 \\
Choroidal effusion & 2 & 1.9 \\
New CSMO & 1 & 0.9 \\
Exacerbation of preexisting CSMO & 2 & 1.9 \\
Anterior uveitis & 2 & 1.9 \\
Transient reduction in visual acuity & 2 & 1.9 \\
Total & 15 & 14
\end{tabular}

$\mathrm{CSMO}=$ clinically significant macular oedema; $\mathrm{PRP}=$ panretinal photocoagulation.

Fifteen patients $(14.0 \%)$ returned with adverse events within the first 8 weeks of treatment. (Table 3) One developed a spreading extramacular TRD requiring vitrectomy, and another, who presented with high-risk proliferative retinopathy, developed a macular TRD. One patient with florid disc neovascularisation developed mild, non-progressive macular tractional folds following PRP, with a reduction of VA from 6/9 to 6/12. Two patients developed new vitreous haemorrhages, one in a patient with preexisting $\mathrm{VH}$ who did not require vitrectomy, and another in a patient who went on to vitrectomy surgery. In one patient, PRP exacerbated an underlying neurotrophic keratopathy, resulting in a persistent epithelial defect with secondary corneal scarring. Two patients developed symptomatic but transient choroidal effusions. The first presented with a drop in acuity from 6/12 to 6/18 on the day after laser, and was noted to have an inferior choroidal effusion. At 2-week review, this had completely resolved. The second noticed a shadow in the temporal field of vision the day after laser treatment, but with no reduction in acuity.

Circumferential peripheral choroidal effusions were present, which were larger nasally. One week later, the patient was no longer symptomatic, with a small residual nasal effusion. One patient developed new CSMO, which resolved spontaneously within 8 weeks. Two further patients experienced exacerbations of preexisting CSMO, though both of these improved spontaneously and did not require treatment. Two patients experienced a transient drop in VA, without $\mathrm{VH}$ or clinical evidence of macular thickening. The first noticed a reduction in VA immediately after PRP and presented with a VA of $6 / 24$. A review was planned in 2 weeks, but he delayed reattendance for 8 weeks, by which time his vision had returned to the pre-PRP level of $6 / 6$. The second presented with a drop in acuity from $6 / 4$ to $6 / 6$, which began to improve within 8 days. At clinic follow-up, 2 months later, the vision had returned to $6 / 4$. Two
Table 4 Nine months follow-up data

\begin{tabular}{|c|c|c|c|}
\hline & $M P H$ & NDRLTA & P-value \\
\hline \multicolumn{4}{|l|}{ Follow-up } \\
\hline Median (months) & 9.4 & 9.1 & \\
\hline Range (days) & $76-499$ & $76-564$ & \\
\hline SD (days) & 64.5 & 56.6 & \\
\hline \multicolumn{4}{|l|}{ Visual acuity (\%) } \\
\hline $6 / 9$ or better & 72.9 & 54.5 & $<0.01$ \\
\hline $6 / 24$ or worse & 11.2 & 21.9 & 0.03 \\
\hline Severe visual loss ${ }^{a}$ & 3.7 & 2.4 & 0.49 \\
\hline \multicolumn{4}{|l|}{ Neovascular changes (\%) } \\
\hline Disappeared & 47.4 & 38.4 & \\
\hline Regressed & 27.4 & 39.4 & \\
\hline Inactive, fibrotic & 9.5 & 11.8 & \\
\hline Unchanged & 9.5 & 4.9 & \\
\hline Deteriorated & 6.3 & 5.4 & 0.97 \\
\hline \multicolumn{4}{|c|}{ Poor morphological outcome (\%) } \\
\hline New VH & 9.3 & 17.4 & 0.08 \\
\hline New TRD & 2.8 & 4.9 & 0.55 \\
\hline Vitrectomy & 7.5 & 1.5 & 0.02 \\
\hline New rubeosis & 0.9 & 2.9 & 0.43 \\
\hline \multicolumn{4}{|c|}{ Number of treatment sessions (\%) } \\
\hline 1 & 81.3 & 23.8 & \\
\hline 2 or 3 & 18.7 & 52.5 & \\
\hline 4 or 5 & 0 & 21.4 & \\
\hline 6 or more & 0 & 2.5 & \\
\hline
\end{tabular}

$\mathrm{MPH}=$ Musgrove Park Hospital; NDRLTA = National Diabetic Retinopathy Laser Treatment Audit; TRD $=$ tractional retinal detachment; $\mathrm{VH}=$ vitreous haemorrhage.

${ }^{a}$ Visual acuity of $6 / 24$ or better at presentation deteriorating to less than $6 / 60$ at follow-up.

patients developed anterior uveitis, both of which settled within 2 weeks on a short course of topical steroids.

The 9-month follow-up data are detailed in Table 4.

\section{Discussion}

In our study sample, patient demographics were broadly comparable with the national audit, with the exception of a greater proportion of male patients (71.0 vs 57.9\%), which is difficult to explain. In the UK Prospective Diabetes Study of type II diabetics, the ratio of male to female was $3: 2 .^{6}$ A significant association has been shown between male gender and the development of proliferative retinopathy in type I diabetes, but this was not borne out in older-onset diabetics. ${ }^{7,8}$ Our patient cohort also differed in baseline best-recorded Snellen VA, with $75.7 \%$, achieving an acuity of $6 / 9$ or better, compared with $55.4 \%$ in the national audit $\left(P<0.01, \chi^{2}\right)$.

Intention to complete treatment in one session $(100 \%$ $\mathrm{MPH}$ vs $41.2 \%$ NDRLTA) is a principal motivation for performing indirect PRP in theatre. Multiple 
reattendances are avoided, reducing the risk of retinopathy progression between sessions. PDR is potentially sight threatening, and we feel the benefits of early completion of treatment outweigh the potential risks of GA, in a procedure which poses very little metabolic challenge. Admission of diabetics on the day of surgery for even major procedures is now common, feasible, and safe. ${ }^{9}$

For patients with concurrent maculopathy, the DRS suggested that applying focal macular laser before PRP might reduce the risk of visual loss. ${ }^{10}$ The majority of our study patients received focal laser before PRP (56.3\%), whereas in the national audit, most patients requiring focal laser were treated at the same time as PRP (45.3\%). In $18.7 \%$ of our patients with concurrent maculopathy, no treatment was planned, compared with only $5.8 \%$ in the national audit. We took this approach because, particularly in young insulin-dependent patients, macular oedema associated with aggressive peripheral ischaemia may resolve following PRP. ${ }^{4}$

Controversy exists as to whether PRP treatment complications differ depending on whether administered in a single session, or divided into multiple sessions spaced over time. ${ }^{11}$ Those who advocate multiple treatment sessions believe that certain adverse effects may be related to placing a large number of laser burns in one sitting. Transient exudative retinal detachment and choroidal detachment are common after argon PRP, but are often only detectable in the immediate posttreatment period. ${ }^{12}$ Although their incidence is higher in eyes treated in a single session, spontaneous resolution occurs within a short period of time, with no long-term sequelae. ${ }^{11}$ A randomised controlled trial published in 1982 showed no difference in the incidence of macular oedema between single and multiple treatment groups at 6 months, and no difference in the incidence of TRD or new $\mathrm{VH}$ over the same time period. ${ }^{11}$ The Diabetic Retinopathy Clinical Research Network is currently conducting an observational study of the development of macular oedema following scatter laser photocoagulation delivered either in one sitting, or in four sittings each separated by 4 weeks, and this is anticipated to provide a more definitive answer to the question.

Of the 15 adverse events that presented within the first 8 weeks of PRP, 10 were mild or transient, resolving within a further 2 months. However, five (4.7\%) were persistent and visually significant. Three of these required vitrectomy during the study period (one macular TRD, one extramacular TRD, and one VH). Despite being well-recognised complications of PRP, these particular events may also be attributed to the natural history of the disease process, especially in the setting of high-risk proliferative retinopathy at presentation. ${ }^{13}$
There was no significant difference in VA change ( $P=0.119$, Mann-Whitney), or the development of severe visual loss between our series and the NDRLTA $(P=0.49$, $\chi^{2}$ ). At 9-month follow-up, $12.1 \%$ of our patients had developed CSMO, which was not present at baseline. This is broadly similar to rates of 6 and $17 \%$ reported in other studies. ${ }^{14,15}$ The rates of new $\mathrm{VH}$, new TRD, and new rubeosis in this study were lower than the national audit, but the vitrectomy rate was significantly higher (7.5 vs $1.5 \%, P=0.02 \chi^{2}$ ). This is partly due to our policy of performing early vitrectomy, and also of performing initial PRP in high-risk cases at presentation, prior to a planned vitrectomy. Furthermore, the national audit vitrectomy rate of $1.5 \%$ was surprisingly low, considering a $17.4 \%$ incidence of new $\mathrm{VH}$ and a $4.9 \%$ incidence of new TRD. This would imply that only a minority of patients who developed new VH or TRD went on to surgery. In another large study comprising 297 patients, the vitrectomy rate at 1 year was $10 \%$, which is comparable to our rate of $7.5 \%$ at 9.4 months. ${ }^{14}$ The timing of vitrectomy continues to be controversial, and the respective roles of continued observation $v s$ augmentation laser therapy or early vitrectomy have not yet been clearly defined. ${ }^{15}$

The limitations of this study include all those associated with a retrospective study. Lack of a randomised control group makes direct comparison of data difficult, and occasionally, essential case note data are missing. However, clinical definitions and the data collection proforma were modelled on the UK national audit as far as possible, and the large sample size allows for a useful evaluation of adverse events.

In conclusion, the visual and morphological outcomes after indirect PRP in our unit were not inferior to the outpatient-based national audit, and the incidence of significant PRP-induced adverse events was low. For patients with PDR, routine, single session, indirect PRP in an operating theatre setting is an acceptable and well-tolerated alternative to slit-lamp-based treatment.

\section{References}

1 Mizuno K. Binocular indirect argon laser photocoagulator. Br J Ophthalmol 1981; 65: 425-428.

2 Gurelik G, Coney JM, Zakov N. Binocular indirect panretinal laser photocoagulation for the treatment of proliferative diabetic retinopathy. Ophthalmic Surg Lasers Imaging 2004; 35: 94-102.

3 Wu W-C, Hsu K-H, Chen T-L, Hwang Y-S, Lin K-K, Li L-M et al. Interventions for relieving pain associated with panretinal photocoagulation: a prospective randomized trial. Eye 2006; 20: 712-719.

4 Bailey CC, Sparrow JM, Grey RH, Cheng H. The national diabetic retinopathy laser treatment audit. II. proliferative retinopathy. Eye 1998; 12: 77-84. 
5 Bailey CC, Sparrow JM, Grey RH, Cheng H. The national diabetic retinopathy laser treatment audit III clinical outcomes. Eye 1999; 13: 151-159.

6 UK Prospective Diabetes Study. IV. Characteristic of newly presenting type 2 diabetic patients: male preponderance and obesity at different ages. Diabet Med 1988; 5: 154-159.

7 Klein R, Klein BE, Moss SE, Davis MD, DeMets DL. The wisconsin epidemiologic study of diabetic retinopathy III prevalence and risk of diabetic retinopathy when age at diagnosis is 30 or more years. Ophthalmology 1984; 102: 527-532.

8 Klein R, Klein BE, Moss SE, Davis MD, DeMets DL. The wisconsin epidemiologic study of diabetic retinopathy II prevalence and risk of diabetic retinopathy when age at diagnosis is less than 30 years. Arch Ophthalmol 1984; 102: 520-526.

9 Robertshaw HJ, McAnulty GR, Hall GM. Strategies for managing the diabetic patient. Best Pract Res Clin Anesthesiol 2004; 18: 631-643.
10 Ferris FL, Podgor MJ, Davis MD. Macular oedema in diabetic retinopathy study patients. Diabetic Retinopathy Study report number 12. Ophthalmology 1987; 94: 754-760.

11 Doft BH, Blankenship GW. Single vs multiple treatment sessions of argon laser panretinal photocoagulation for proliferative diabetic retinopathy. Ophthalmology 1982; 89: 772-779.

12 Liang JC, Huamonte FU. Reduction of immediate complications after panretinal photocoagulation. Retina 1984; 4: 166-170.

13 McDonald HR, Schatz H. Visual loss following panretinal photocoagulation for proliferative diabetic retinopathy. Ophthalmology 1985; 92: 388-393.

14 Kaiser RS, Maguire MG, Grunwald JE, Lieb D, Jani B, Bruker AJ et al. One-year outcomes of panretinal photocoagulation in proliferative diabetic retinopathy. Am J Ophthalmol 2000; 129: 178-185.

15 Reddy VM, Zamora RL, Olk RJ. Quantitation of retinal ablation in proliferative diabetic retinopathy. $A m \mathrm{~J}$ Ophthalmol 1995; 119: 760-766. 